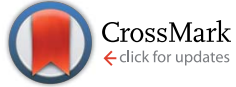

Cite this: RSC Adv., 2016, 6, 55159

Received 28th March 2016 Accepted 31st May 2016

DOI: 10.1039/c6ra08030h

www.rsc.org/advances

\section{Hierarchical structured polymers for light- absorption enhancement of silicon-based solar power systems $\uparrow$}

\begin{abstract}
Jung Woo Leem, \$Minkyu Choi, \$ Bhaskar Dudem and Jae Su Yu*
Light-absorption enhancement of silicon (Si)-based solar power systems (i.e., solar modules) is reported by employing a ultraviolet-curable polymer (i.e., NOA63) film integrated with a hierarchical structure (HS) consisting of nanonipples on micropyramids onto the PET cover as a protective antireflection (PAR) layer. The HS-patterns can be easily transferred from Si molds with the HS via poly-dimethylsiloxane stamps by soft lithography. The HS-NOA63 film increases the total and diffuse transmittances of the bare polyethylene terephthalate (PET), simultaneously, at wavelengths of 380-1100 nm, thus resulting in the reduced surface reflection (i.e., lower solar weighted reflectance $\left(R_{\mathrm{sw}}\right)$ of $6.1 \%$ ) and the strong light scattering (i.e., much higher average haze ratio $\left(H_{\text {avg }}\right)$ of $90.7 \%$ ) compared to the bare PET (i.e., $R_{\mathrm{sw}}=$ $11.7 \%$ and $H_{\text {avg }}=6.9 \%$ ). Consequently, the Si solar modules with the HS-NOA63 PAR layer of the PET cover exhibit a superior solar power generation in a wide incident angle range $\left(0-70^{\circ}\right)$ of solar irradiance, especially, showing a relative increment percentage of $6.3 \%$ in power conversion efficiency (PCE) at $0^{\circ}$ (i.e., from PCE $=13.46$ to $14.3 \%$ ). Also, it has a superhydrophobic surface with a high water contact angle of $150^{\circ}$, which leads to a self-cleaning ability to protect Si solar modules from being polluted by water droplets and dust particles in outdoor applications.
\end{abstract}

\section{Introduction}

Polyethylene terephthalate (PET) has been widely employed as device substrates or protection covers in various optoelectronic and electronic applications such as solar power systems, displays, transistors, and triboelectric generators due to its flexibility, lightweight, low cost, high tolerance, and transparency. ${ }^{1-4}$ Particularly, the PET has been generally used to protect the solar power systems (i.e., solar cells or modules/ panels), which are mostly exposed to external shock, heat, ultraviolet (UV) radiation, and corrosive acidic rain in real outdoor environments. ${ }^{5,6}$ But, the PET with a refractive index $(n)$ of 1.65 exhibits the relatively lower transmission of $88 \%$ for both sides (i.e., reflection of $6 \%$ for one side) compared to those (i.e., transmission $\geq 92 \%$ ) of other transparent substrates or covers including glasses and polymers (i.e., $n \leq 1.5$ ). This low transmission can be one of deterioration factors of the

Department of Electronics and Radio Engineering, Kyung Hee University, 1732 Deogyeong-daero, Giheung-gu, Yongin-si, Gyeonggi-do 17104, South Korea. E-mail: jsyu@khu.ac.kr

$\dagger$ Electronic supplementary information (ESI) available: Photograph of a water droplet for the flat NOA63 film on the PET (F-NOA63:PET), sequential photographs of the water droplet cleaning behaviours for the reference Si solar module with the bare PET cover, and reproducibility of HS-NOA63:PET samples on optical properties for different RHS-PDMS stamps via one HS-Si mold. See DOI: $10.1039 / \mathrm{c} 6 \mathrm{ra} 08030 \mathrm{~h}$

\$ These authors contributed equally to this work. performance in solar power systems. Thus, an efficient light-harvesting layer such as antireflection coatings (ARCs) on the outer surface of PET cover is essential to enhance the transmission. Recently, hierarchical architectures with a combination of subwavelength-scale nanostructures and lens-like or tapered microstructures effectively not only further suppress the surface reflection, but also lead to the stronger light scattering in the transmission over wide ranges of light incident wavelengths and angles compared to other ARCs such as the nanostructures and thin multilayers on the flat surface. ${ }^{7-12}$ However, the directlypatterned hierarchical structures on substrates or covers were usually fabricated by nano/micro-lithography patterning (i.e., electron-beam lithography, nanoimprint lithography, photolithography, colloidal lithography, etc.) and subsequent dry etching processes, thus increasing the complexity and cost of the fabrication process including a size limitation. Therefore, it is required to develop efficient ARCs of PET covers in a relatively inexpensive, simple, and high-throughput method.

Soft lithography, which is widely utilized to form nano/ microstructures onto a pattern-transferable polymer surface of substrates or covers from master molds in a variety of applications such as optoelectronics, fluidic mechanics, and biologics because of its simple, fast, and inexpensive process as well as high throughput, can be an effective alternative fabrication approach. ${ }^{\mathbf{1 3 - 1 6}}$ Although the master molds are also fabricated by nano/micro-lithography patterning and etching processes, once both the molds and replica stamps are made, they can be 
repeatedly used for pattern transfers into secondary substrates or covers. Besides, the soft lithography process (e.g., roll-to-roll or roll-to-plate via large-scale silicon ( $\mathrm{Si}$ ) wafers with diameters of $\geq 4$ inch as a master mold) can be also suitable for mass-productive industry applications. ${ }^{17,18}$

Over the past years, to reduce the surface reflection, many studies have been reported on hierarchical structured polymers with low refractive indices such as poly-dimethylsiloxane (PDMS), polycarbonate, and polyurethane, ${ }^{19-21}$ but these polymers have the optical absorption at wavelengths larger than $800 \mathrm{~nm}$, which might be not appropriate for low band gap material-based solar cells (i.e., Si, GaAs, etc.) with broad wavelength absorption bands. ${ }^{22,23}$ Whereas, a urethane-based UV-curable Norland Optical Adhesive 63 (NOA63) can be an proper polymer as an ARC layer of PET cover because of its lower $n$ value of $\sim 1.56$ compared to the PET $(n \sim 1.65) .{ }^{24,25}$ Moreover, it has almost no absorption over a wide wavelength range of $400-1100 \mathrm{~nm}$ in solar irradiance. ${ }^{26}$ In addition, it exhibits strong mechanical strengths, such as high modulus ( $>1.6 \mathrm{GPa}$ ), hardness, robustness, and flexibility, ${ }^{27,28}$ which facilitate the nano/micro-scale patterns on its surface via soft lithography. It is also reported that there is no considerable discoloration or deterioration of the NOA films, which were tested on UV exposure systems consisting of a rotating turntable and four $275 \mathrm{~W}$-sunlamps positioned 10 inch away for 7 weeks and heattreated in an oven at temperatures of $25-160{ }^{\circ} \mathrm{C} .^{26,29}$ Meanwhile, a self-cleaning function on the surface of ARCs is very useful to wash any pollutants on the surface of solar power systems in real outdoor environments. As a result, it helps to maintain good photovoltaic (PV) performance of solar power systems for a long period of time. ${ }^{30-32}$

Several studies have reported on the ARCs of PET such as motheye structured UV resist, porous or low- $n$ thin-film multilayers, and microstructured NOA63 polymer. ${ }^{33-35}$ However, there are very few or no reports on the use of hierarchical structures consisting of nanonipples on micropyramidal arrays at the surface of polymers with the multifunctions (i.e., AR, light scattering, and self-cleaning) for a protection of PET covers in solar power systems via soft lithography. Herein, to fabricate the Si master molds with hierarchical structures, the relatively simple, facile, and cost-effective process techniques were utilized including the potassium hydroxide ( $\mathrm{KOH})$-based chemical wet etching process for the microstructures (i.e., micropyramidal arrays) and thermallydewetted metal nanoparticles as an etch mask for the nanostructures with nipple arrays. Besides, both the approaches also enable to form the hierarchical structures on the surface of largearea Si master molds (e.g., Si wafers with diameters of $\geq 4$ inch). ${ }^{36-38}$ Therefore, it is very meaningful to study the PV performance of solar power systems incorporated with a hierarchical structured protective antireflection (PAR) polymer layer on the outer surface of PET cover, including their optical and surface wetting behaviours. In this work, we fabricated the artificial hierarchical structure (HS) consisting of nanonipples on micropyramidal arrays on the surface of NOA63 films by the soft lithography via Si molds with the HS prepared from wet etching and thermally-dewetted gold $(\mathrm{Au})$ nanopatterning processes. Their water wettability (i.e., self-cleaning ability) and optical properties, together with a theoretical analysis of optical light-scattering properties by a finite- difference time-domain (FDTD) simulation, were studied. The influence of HS-patterned NOA63 films as a PAR layer of PET covers was explored on the PV performance of Si-based solar power systems (i.e., solar modules). Their incident sunlight angle-dependent PV performance was also investigated.

\section{Experimental and simulation details}

\section{Fabrication of samples}

Fig. 1 shows the schematic illustration of hierarchical (i.e., nano/micro) pattern-transfer steps at the surface of NOA63 films on the PET cover from Si molds via soft lithography. The scanning electron microscope (SEM) images of the corresponding structures for the fabrication of Si molds are also shown in Fig. 1(a)-(c). Prior to the preparation of Si molds, for the removal of oxide and organic contaminants on the $\mathrm{Si}$ surface, the Si samples were cleaned by acetone, methanol, and de-ionized (DI) water in an ultrasonic cleaning bath for $10 \mathrm{~min}$, and then they also dipped into to buffered oxide etchant, $5 \mathrm{wt} \%$ hydrofluoric acid solution, and DI water for $1 \mathrm{~min}$, sequentially. Firstly, to form a hierarchical structure consisting of nanonipples on micropyramidal arrays on the Si surface, the cleaned Si samples were etched in aqueous solutions mixed by the $\mathrm{KOH}$, isopropyl alcohol, and DI water ( $1: 0.5: 8.5 \mathrm{vol} \%)$ at a temperature of $75{ }^{\circ} \mathrm{C}$ for $40 \mathrm{~min}$, followed by the treatment in a hydrogen chloride, hydrogen peroxide, and DI water ( $\mathrm{HCl}: \mathrm{H}_{2} \mathrm{O}_{2}: \mathrm{H}_{2} \mathrm{O}=1: 1: 5$ ) mixture solution at $80{ }^{\circ} \mathrm{C}$ for $10 \mathrm{~min}$, which can remove the remained potassium impurities on the etched Si surface, thus creating the microstructured $\mathrm{Si}$ (MS-Si) with pyramidal arrays (i.e., average height $=2 \pm 1 \mu \mathrm{m}$, average period $=3 \pm 1.5 \mu \mathrm{m}$ ), as shown in the SEM image of Fig. 1(a). After that, for the nanostructure (NS), the Au thin film with a thickness of $5 \mathrm{~nm}$ was deposited on the surface of MS-Si samples by using a thermal evaporation system. To form the dot-like nanoparticles (NPs) as an etch mask pattern, the samples were heat-treated by using a rapid thermal annealing system at a temperature of $600{ }^{\circ} \mathrm{C}$ for $2 \mathrm{~min}$ in

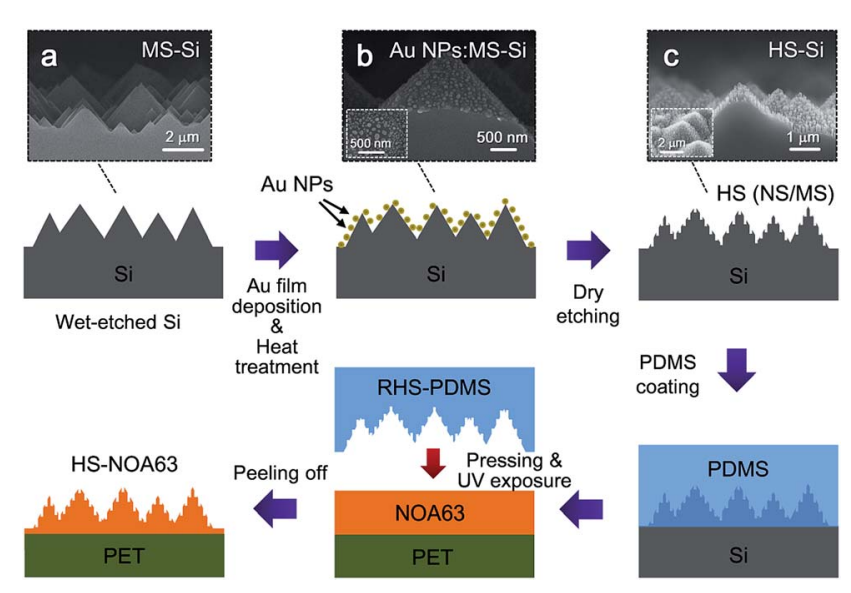

Fig. 1 Schematic illustration of hierarchical (i.e., nano/micro) patterntransfer steps at the surface of NOA63 films on the PET cover from Si molds via soft lithography. SEM images of the corresponding structures for the fabrication of Si molds are also shown in $(a-c)$. 
a nitrogen environment, indicating the average size and period of $80 \pm 20 \mathrm{~nm}$ and $200 \pm 80 \mathrm{~nm}$ for Au NPs, respectively. Using the thermally-dewetted Au NPs (Fig. 1(b)), the Si molds with the hierarchical structure (i.e., HS-Si) consisting of nanonipples on micropyramidal arrays were fabricated by an inductively coupled plasma (ICP) etching process (Fig. 1(c)), showing the average height and period of $280 \pm 20 \mathrm{~nm}$ and $200 \pm 80 \mathrm{~nm}$ for nanonipples on the MS-Si, respectively. The ICP etching was carried out under RF power of $25 \mathrm{~W}$ and ICP power of $100 \mathrm{~W}$ in $\mathrm{CF}_{4}$ : Ar $(20: 60 \mathrm{sccm})$ gas flow at $5 \mathrm{mTorr}$ for $15 \mathrm{~min}$. To transfer the HS-patterns (i.e., nanonipples/micropyramidal arrays) on Si molds onto the surface of NOA63 films, PDMS stamps were fabricated. A hard PDMS mixture solution (VDT-731, SIP 6831.1, SIT7900, and HMS-301, Gelest Inc.) was spin-coated on Si molds, and then the samples were thermally cured in an oven. As a nondestructive and reversible manner, to enable manual application and intimate conformable contact on flat surfaces, a soft PDMS (base resin and curing agent, weight ratio $=10: 1$ ) was covered on the hard PDMS : Si mold and cured again. Lastly, the reversely HS-patterned PDMS (RHS-PMDS) stamp was obtained by separating the PDMS from the Si mold. The NOA63 film was spin-coated on PET covers at $3000 \mathrm{rpm}$ for $1 \mathrm{~min}$, and then immediately the samples were pressed with $3.0 \mathrm{kgf}$ by the RHS-PDMS stamp. After UV exposure for $20 \mathrm{~min}$, the $\mathrm{HS}$ on the surface of NOA63:PET (i.e., HS-NOA63:PET) was formed by peeling off the RHS-PDMS stamp. For comparison, the flat NOA63 (F-NOA63), NS-NOA63, and MS-NOA63 samples were also fabricated on PET covers using the same pattern-transfer processes, respectively. Finally, for practical device applications, all the fabricated NOA63 films were employed onto the PET cover of commercial available Si solar modules consisting of 2-cells with an area of $2.7 \times 2.7 \mathrm{~cm}^{2}$ as a PAR layer. The Si solar cells in modules were completely encapsulated on a printed circuit board (PCB), together with an ethylene vinyl acetate (EVA) adhesive and a PET cover.

\section{Characterization instruments}

The etched and surface profiles of the fabricated samples were observed by using a field-emission SEM system (LEO SUPRA 55, Carl Zeiss). The optical properties were characterized by using a UV-vis-NIR spectrophotometer (Cary 5000, Varian) with an integrating sphere. The water contact angles were measured and averaged at three different positions on the surface of the samples by using a contact angle measurement system (Phoenix-300, SEO Co., Ltd.) with a $5 \mu \mathrm{L}$ droplet of DI water. The current-voltage $(I-V)$ characteristics of Si solar modules were measured by using a photocurrent system consisting of a solar simulator (ABET, SUN 3000) with $1000 \mathrm{~W}$ Xe short arc lamp and a source meter (Keithley 2400) under 1-sun air mass 1.5 global (AM1.5g, $100 \mathrm{~mW} \mathrm{~cm}^{-2}$ ) illumination at room temperature. For angle-dependent PV performance measurements, inclined mount holders with different tilting angles of $20-70^{\circ}$ were used.

\section{Numerical modelling and simulations}

The theoretical analysis of the light-scattering property for the HS-NOA63:PET was performed using the FDTD method. To design the simulation model, hierarchical nanonipples (i.e., NS) on the micropyramidal arrays (i.e., MS) were roughly represented by a periodic geometry with a triangular pyramid shape for both the NS and MS in the Cartesian coordinate system of a scalar-valued function of two variables, $f(x, z)$, for simplicity. The incident light entered from air into the structure at normal incidence. The amplitude of $y$-polarized electric field, i.e., $E_{y}$, was calculated for the incident plane wave with a slab mode beam profile in the continuous-wave mode, which was normalized at a wavelength $(\lambda)$ of $525 \mathrm{~nm}$. The height/period of NS and MS on the NOA63 film were kept at $250 \mathrm{~nm} / 200 \mathrm{~nm}$ and $2 \mu \mathrm{m} / 3 \mu \mathrm{m}$, respectively. The thicknesses of NOA63 and PET films were set to be 10 and $200 \mu \mathrm{m}$, respectively. The refractive indices of the PET and NOA63 used in these calculations were assumed to be 1.65 and 1.56 , respectively.

\section{Results and discussion}

\section{Surface morphologies and patterned profiles}

Fig. 2 shows the SEM images of (a) NS-, (b) MS-, and (c) HSNOA63 films on PET covers. The high-magnification SEM image of HS-NOA63 is shown in Fig. 2(d). Using the soft lithography, the tapered micropyramidal arrays (i.e., MS) were well formed on the surface of the NOA63 film, as can be seen in Fig. 2(b). For the MS, the average height and period were roughly estimated to be $2 \pm 1 \mu \mathrm{m}$ and $3 \pm 1.5 \mu \mathrm{m}$, respectively. Also, the nanonipples (i.e., NS) were relatively well transferred on both the flat and MS surfaces of NOA63 films (Fig. 2(a) and (c)) from the corresponding Si molds via PDMS stamps without significantly large pattern deformations, indicating the average height and period of $250 \pm 50 \mathrm{~nm}$ and $200 \pm 80 \mathrm{~nm}$, respectively, for the nanonipples. Here, it is noteworthy that the HS comprised of a combination of nanonipples and micropyramidal arrays can be easily realized on the wanted secondary surface from the HS-Si mold prepared by the simple and costeffective processes such as wet-chemical solution etching for the MS and thermally-dewetted metal nanoparticles as an etch mask for the NS without any complicated and expensive nano-
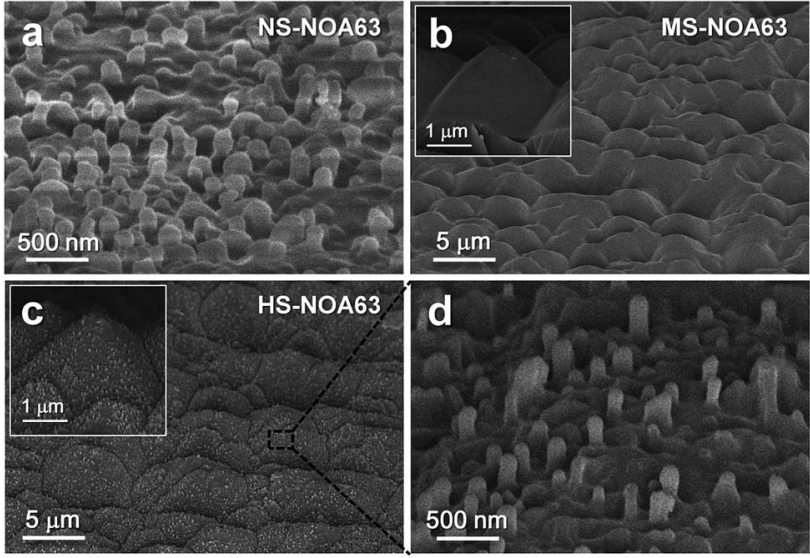

Fig. 2 SEM images of (a) NS-, (b) MS-, and (c) HS-NOA63 films on PET covers. (d) High-magnification SEM image of the HS-NOA63. 
and microlithography processes such as e-beam lithography, nanoimprint lithography, and photolithography, to form nanoand micropatterned etch masks. These processes are also able to make mass production because of their large-scalable fabrication as well as the advantages (i.e., simplicity, low cost, high throughput, etc.) of soft lithography, as mentioned above.

\section{Optical properties}

Fig. 3 shows the total (a) transmittance and (b) reflectance spectra of F-, NS-, MS-, and HS-NOA63 films on PET covers. For comparison, the total transmittance and reflectance spectra of bare PET are also shown in Fig. 3(a) and (b), respectively. In Fig. 3(a), it can be observed that the transmittance of the PET was improved over a wide wavelength range of 380-1100 $\mathrm{nm}$ by coating the NOA63 film (i.e., F-NOA63:PET), exhibiting the increased average total transmittance $\left(T_{\text {avg }}\right)$ value of $89.6 \%$ (i.e., $T_{\text {avg }}=88.6 \%$ for the bare PET). This is attributed to the step refractive index distribution of constituent materials (i.e., air/ NOA63/PET $=1 / 1.56 / 1.65) .{ }^{39-43}$ On the other hand, the introduction of NS into the flat NOA63 film led to the further increase
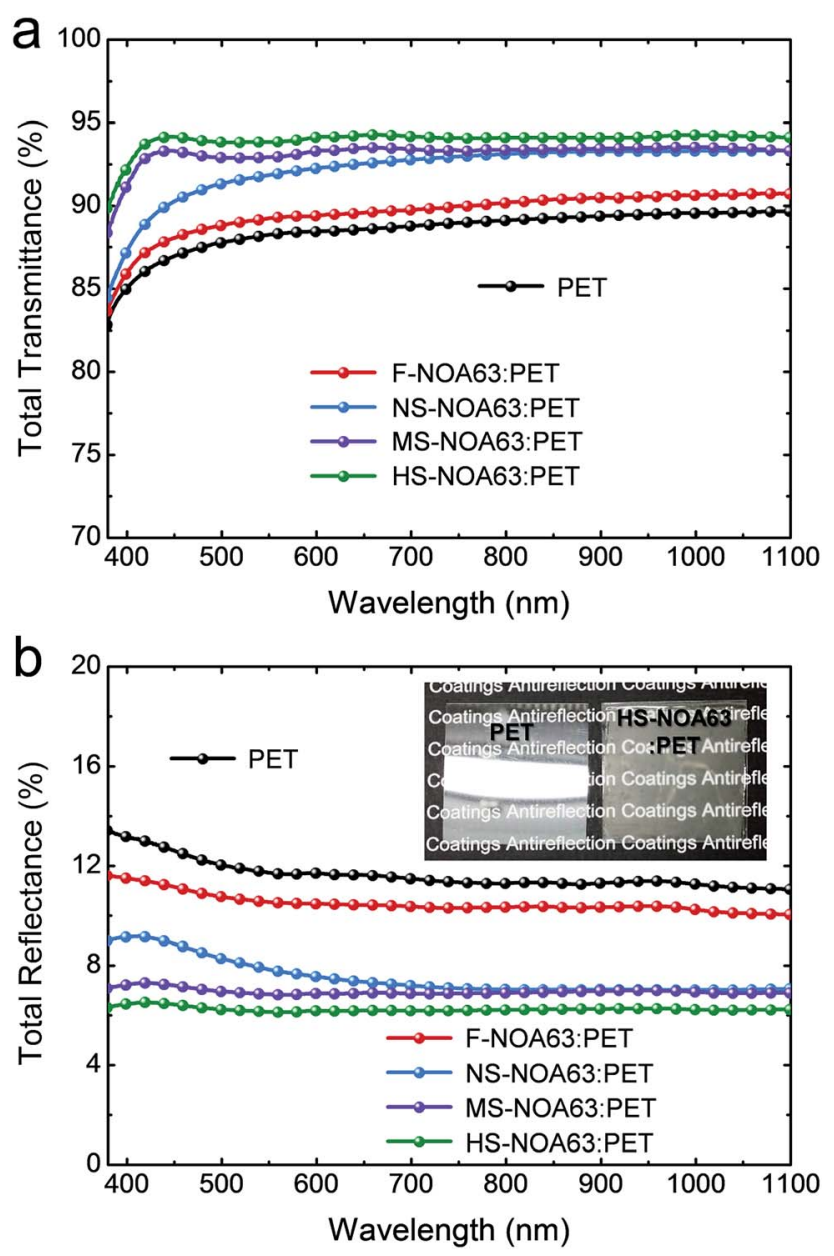

Fig. 3 Total (a) transmittance and (b) reflectance spectra of F-, NS-, MS-, and HS-NOA63 films on PET covers. For comparison, both the total transmittance and reflectance spectra of bare PET are also shown in (a) and (b), respectively. in the transmittance (i.e., $T_{\mathrm{avg}}=92.3 \%$ ) due to the additional formation of the linearly and continuously gradient effective refractive index profile from air to the NOA63 film, caused by the nanonipples. ${ }^{39-41}$ However, both the MS-NOA63:PET and HSNOA63:PET with micropyramidal arrays had higher transmittance spectra compared to the other samples. This is because the microstructures with a tapered pyramidal geometry have a more linearly gradient effective refractive index distribution between air and the NOA63 film owing to the relatively much larger height of $2 \pm 1 \mu \mathrm{m}$ and also lead to the extension of effective optical path lengths caused by the diffracted and rebounded lights between the micropyramids, which can efficiently increase the transmission in transparent materials by decreasing the surface reflectance (see Fig. S1 of the ESI $\dagger$ ). ${ }^{\mathbf{4 3 , 4 4}}$ Especially, the HS-NOA63:PET with the NS (i.e., nanonipples) showed the higher $T_{\mathrm{avg}}$ value of $94 \%$ than that (i.e., $T_{\mathrm{avg}}=$ 93.2\%) of the MS-NOA63:PET. This is the reason why the nanonipples with subwavelength-scale periods shorter than incident light wavelengths not only eliminate the higher diffracted orders and thus allow only zeroth order, but also form the more linearly gradient effective refractive index profile from air to the MS-NOA63. ${ }^{39-41}$ As can be seen in Fig. 3(b), the reflectance spectra also showed a similar tendency to the total transmittance data of Fig. 3(a). The HS-NOA63:PET had the lower average total reflectance $\left(R_{\mathrm{avg}}\right)$ value of $6.2 \%$ compared to the other samples (i.e., $R_{\mathrm{avg}}=11.6,10.5,7.7$, and $6.9 \%$ for the PET, F-NOA63:PET, NS-NOA63:PET, and MS-NOA63:PET, respectively). The superior antireflection effect of HS-NOA63 can be verified from the photograph in the inset of Fig. 3(b). For the bare PET without the NOA63 film and any patterns, the characters under it were nearly not seen due to the strongly reflected white florescent light at the surface. On the contrary, the HS-NOA63:PET exhibited a relatively better legibility for the characters below the sample. For PV applications, it is also crucial to estimate the solar spectrum weighted optical property, which is the ratio of the usable photons transmitted (reflected) to the total usable photons, of samples. ${ }^{42}$ As expected, the HS-NOA63:PET also had the higher (lower, for the reflectance) solar weighted transmittance $\left(T_{\mathrm{sw}}\right)$ value of $94.1 \%\left(R_{\mathrm{sw}}=\right.$ $6.1 \%$ ) compared to the other samples (i.e., $T_{\mathrm{sw}}=88.5,89.5,92.1$, and $93.3 \%$ and $R_{\mathrm{sw}}=11.7,10.6,7.9$, and $6.8 \%$ for the PET, FNOA63:PET, NS-NOA63:PET, and MS-NOA63:PET, respectively). Therefore, the HS-NOA63 film with a low $n$ value of 1.56 can further effectively suppress the surface reflection, thus resulting in the enhanced transmission in transparent substrates or covers. For the reproducibility in soft lithography, five HS-NOA63:PET samples or more for each RHS-PDMS stamp were fabricated from one HS-Si mold and their optical properties were investigated (see Table $\mathrm{S} 1$ of the ESI $\dagger$ ). For all the HS-NOA63:PET samples from no. 1 to no. 5 prepared by each RHS-PDMS stamp, the $T_{\text {avg }}$ and $T_{\mathrm{sw}}$ values were maintained at $94 \%$ (standard deviation error range $= \pm 0.38 \%$ ) and $94.1 \%$ $( \pm 0.36 \%)$, respectively. On the other hand, for the RHS-PDMS stamp (no. 1), the $T_{\mathrm{avg}}$ and $T_{\mathrm{sw}}$ values of HS-NOA63:PET samples at numbers larger than 6 (i.e., no. 6-8) were generally decreased, indicating $T_{\text {avg }}=93.77 \pm 0.19 \%$ and $T_{\mathrm{sw}}=93.88 \pm$ $0.2 \%$ at no. 8 , which is the 8 times-made HS-NOA63:PET sample 
by the RHS-PDMS stamp (no. 1), respectively. Nevertheless, the absolute decrease percentages of its $T_{\mathrm{avg}}$ and $T_{\mathrm{sw}}$ values are only $0.23 \%$ and $0.22 \%$, respectively. From these results, it can be noted that the HS-patterned NOA63 films have the relatively superior reproducibility via Si molds and PDMS stamps in soft lithography. Thus, considering the large-area roll-to-roll or rollto-plate soft lithography system, the HS-NOA63 films with good optical performance can be repeatedly fabricated on any largesized transparent substrates or covers, resulting in the simple and cost-effective mass production.

The micropyramidal arrays also cause strong light scattering which can enhance the light absorption in the active layer of solar cells. ${ }^{\mathbf{8} 10,11}$ Fig. 4 shows (a) the diffuse transmittance spectra of F-, NS-, MS-, and HS-NOA63 films on PET covers including the bare PET and (b) the contour plots of calculated
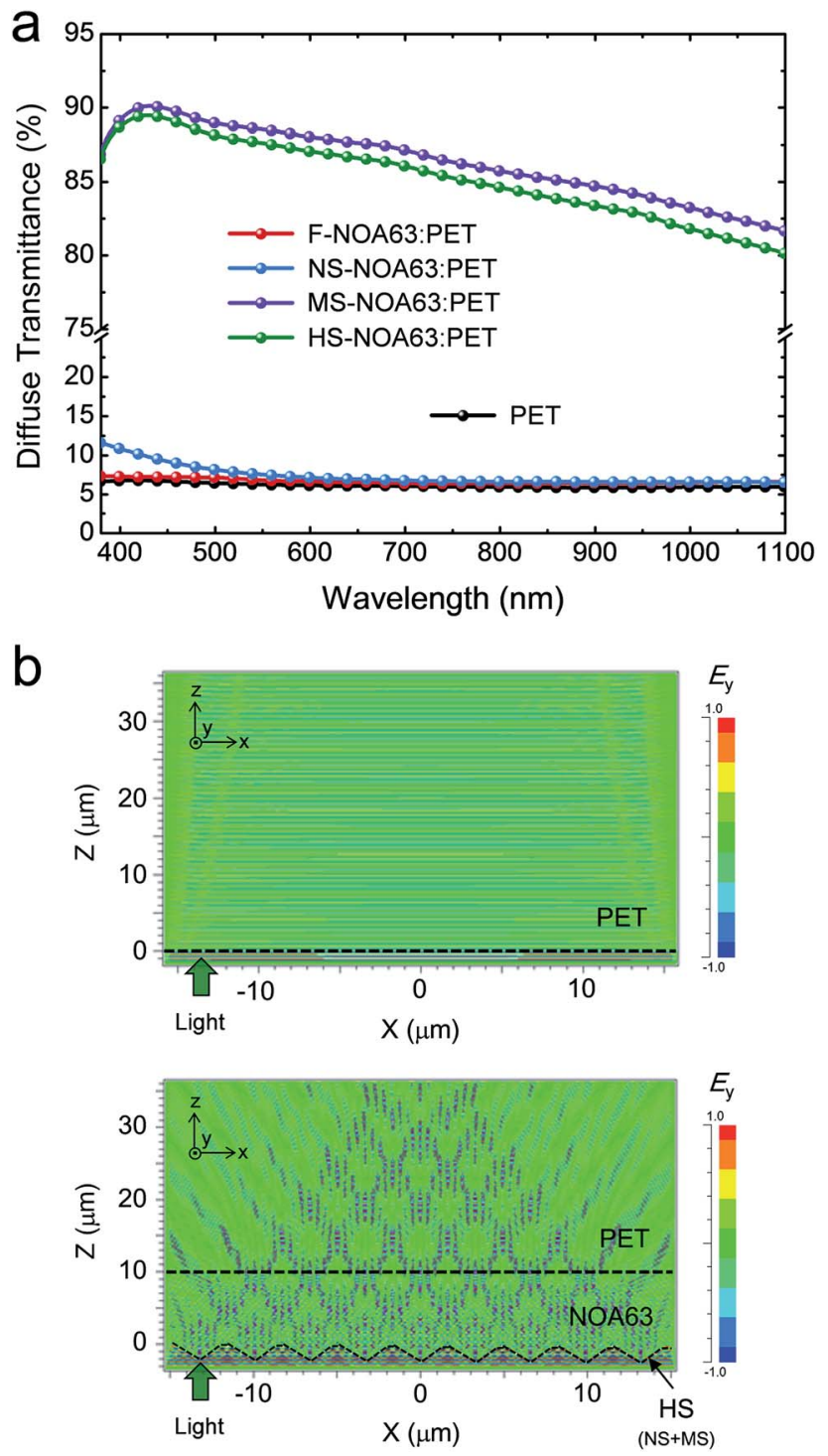

Fig. 4 (a) Diffuse transmittance spectra of F-, NS-, MS-, and HSNOA63 films on PET covers including the bare PET. (b) Contour plots of calculated electric field intensity distributions by FDTD simulations for the incident light propagating from air to the bare PET and HSNOA63:PET, respectively. electric field intensity distributions by FDTD simulations for the incident light propagating from air to the bare PET and HS-NOA63:PET, respectively. As shown in Fig. 4(a), for PET, F-NOA63:PET, and NS-NOA63:PET with a flat surface structure, there are almost no diffused lights, exhibiting diffuse transmittance spectra lower than approximately $10 \%$ at wavelengths of 380-1100 $\mathrm{nm}$. In contrast, both the MS-NOA63:PET and HS-NOA63:PET had large diffuse transmittance spectra $(>80 \%)$. To evaluate the light-scattering property of samples, the haze ratio $(H)$, i.e., $H(\%)=T_{\text {diffuse }} / T_{\text {total }} \times 100$, where $T_{\text {diffuse }}$ and $T_{\text {total }}$ are diffuse and total transmittance spectra, respectively, was calculated. The PET, F-NOA63:PET, and NS-NOA63:PET showed low average $H\left(H_{\text {avg }}\right)$ values of $6.9,7.4$, and $8 \%$ at wavelengths of $380-1100 \mathrm{~nm}$, respectively. On the other hand, for the MS-NOA63:PET and HS-NOA63:PET, much larger $H_{\text {avg }}$ values of 92.6 and $90.7 \%$ were estimated. This is ascribed to the micro-scale pyramid (i.e., grating) structure. However, the HSNOA63:PET showed a relatively lower $H_{\text {avg }}$ value (i.e., $H_{\text {avg }}=$ $90.7 \%$ ) than that (i.e., $H_{\mathrm{avg}}=92.6 \%$ ) of MS-NOA63:PET. In the grating structures with periods larger than incident wavelengths, higher order diffraction lights are generated in transmission. Moreover, the effective optical path lengths are also extended. ${ }^{43,44}$ On the contrary, in the subwavelength-scale nanostructures, the increased transmission with the only zeroth diffraction order can be achieved, thus suppressing the light scattering and increasing the light traveling in a straight line. ${ }^{40,41}$ Therefore, hierarchical structures with a combination of desirable nano- and micro-scale dimensions can enhance both the total and diffuse transmittance spectra over a wide wavelength range, simultaneously, as can be seen in Fig. 3(a) and 4(a), respectively. For the diffraction phenomenon of HS-NOA63:PET, the theoretical analysis was also carried out via the FDTD method. In Fig. 4(b), for the PET, there is no diffraction in traveling lights, while the HS-NOA63:PET consisting of nanonipples on micropyramidal arrays has strong light interference patterns with a wide angular spread and helps the light propagation across the interface between air and the NOA63. From these optical results, it can be expected that the integration of the HS-NOA63 film onto the outer surface of PET cover in solar modules enables to boost the light absorption in solar cells due to its good light harvesting (i.e., AR and lightscattering effects).

\section{Practicability in Si solar modules}

The device feasibility of the HS-NOA63 film with efficient AR and light-scattering effects was explored on the PV performance of Si solar modules as a PAR layer of the PET cover. Fig. 5 shows the $I-V$ curves of Si solar modules (2-cells) with F-, NS-, MS-, and HS-NOA63 films on PET covers at an incident angle of $0^{\circ}$. For comparison, the $I-V$ curve of the Si solar module with the bare PET cover as a reference is also shown. The PV characteristics of Si solar modules are summarized in Table 1. As shown in Fig. 5, by introducing the NOA63 films onto the PET cover of Si solar modules, the enhanced $I_{\mathrm{sc}}$ values were obtained compared to the reference Si solar module, whereas having similar $V_{\text {oc }}$ and FF values, and hence they increased the PCE of Si solar 


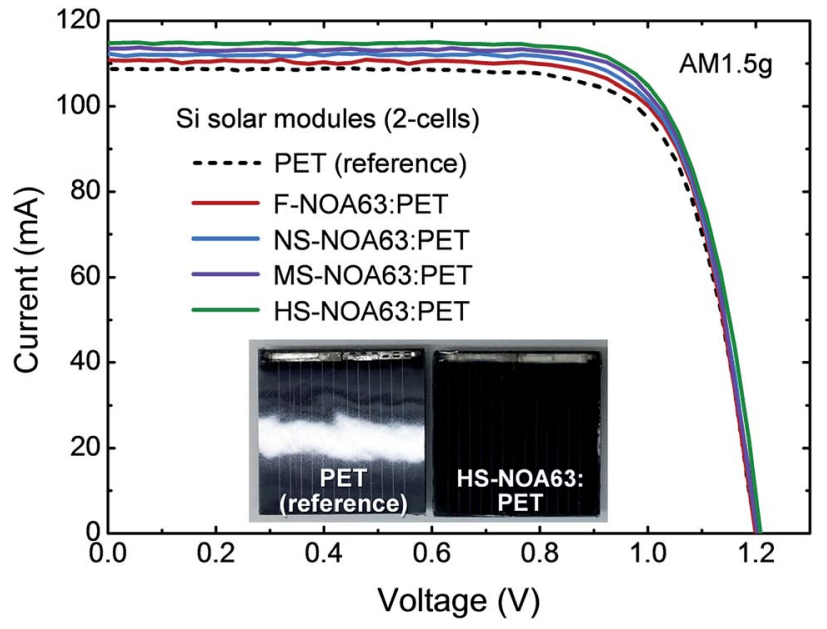

Fig. 5 I-V curves of Si solar modules (2-cells) with F-, NS-, MS-, and HS-NOA63 films on PET covers at an incident angle of $0^{\circ}$. For comparison, the $I-V$ curve of the Si solar module with the bare PET cover as a reference is shown. Photograph of the reference Si solar module and the Si solar module with HS-NOA63:PET is shown in the inset.

modules. Particularly, for the $\mathrm{Si}$ solar module with the HS-NOA63:PET, the larger $I_{\mathrm{sc}}$ value of $114.8 \mathrm{~mA}$ was achieved than those of the other Si solar modules (i.e., $I_{\mathrm{sc}}=108.7,110.7$, 112.2, and 113.5 $\mathrm{mA}$ for PET, F-NOA63:PET, NS-NOA63:PET, and MS-NOA63:PET, respectively). This is attributed to its lower reflectivity (i.e., $R_{\mathrm{sw}}=6.1 \%$ ) and strong light scattering $\left(H_{\text {avg }}=90.7 \%\right)$ over a wide wavelength range of 380-1100 nm, which causes the increased photocurrents in the absorption layer of Si solar cells..$^{\mathbf{8 1 1 , 3 0}}$ As a result, the PCE value was improved from 13.46 to $14.3 \%$, exhibiting the PCE relative increment percentage of approximately $6.3 \%$. The AR effect of the HS-NOA63 film on the Si solar module can be also observed from the photograph in the inset of Fig. 5. The white fluorescent light is strongly reflected at the surface of the reference Si solar module with the bare PET cover while it was almost not reflected at the surface of the Si solar module with the HSNOA63:PET due to the superior AR ability of HS-NOA63 film.

In practical environments, the sunlight incident angle-dependent PV performance is also important because the incident angle of solar irradiance is broad due to the positional variation of the sun in a day and the seasons. Fig. 6 shows the $I_{\mathrm{sc}}$ of Si solar modules with F-, NS-, MS-, and HS-NOA63 films on PET covers including the reference Si solar module and the

Table 1 PV characteristics of Si solar modules

\begin{tabular}{lllll}
\hline Si solar module & $V_{\text {oc }}[\mathrm{V}]$ & $I_{\mathrm{sc}}[\mathrm{mA}]$ & FF [\%] & PCE [\%] \\
\hline Reference (PET) & 1.200 & 108.7 & 75.24 & 13.46 \\
F-NOA63:PET & 1.200 & 110.7 & 75.41 & 13.74 \\
NS-NOA63:PET & 1.201 & 112.2 & 75.18 & 13.90 \\
MS-NOA63:PET & 1.202 & 113.5 & 75.23 & 14.08 \\
HS-NOA63:PET & 1.203 & 114.8 & 75.51 & 14.30
\end{tabular}

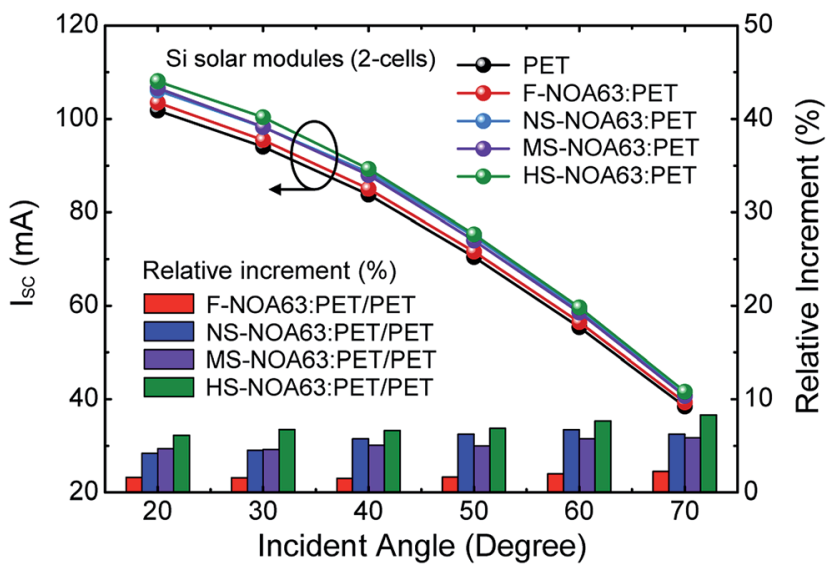

Fig. $6 I_{\mathrm{sc}}$ of Si solar modules with F-, NS-, MS-, and HS-NOA63 films on PET covers including the reference Si solar module and increment percentage in $I_{\text {sc }}$ for the Si solar modules with the corresponding NOA63 films relative to the reference Si solar module at incident angles of $20-70^{\circ}$.

increment percentage in $I_{\mathrm{sc}}$ for the Si solar modules with the corresponding NOA63 films relative to the reference Si solar module at incident angles of $20-70^{\circ}$. For all the Si solar modules, the $I_{\mathrm{sc}}$ values were generally decreased with increasing the incident angle. This is ascribed to the reduction of the projection area where the incident light enters into the Si solar module due to the tilted solar module from the normally incident light source of the solar simulator as well as the increased surface reflection losses. But, the Si solar module with the HSNOA63:PET showed the higher $I_{\mathrm{sc}}$ value at each incident angle, indicating the larger average relative increment percentage of $7.1 \%$ (i.e., HS-NOA63:PET/PET) at $20-70^{\circ}$ compared to the other Si solar modules (i.e., 1.8, 5.6, and $5.2 \%$ for F-NOA63:PET/PET, NS-NOA63:PET/PET, and MS-NOA63:PET/PET, respectively). Thus, the use of the HS-NOA63 film as a PAR layer can effectively enhance the light absorption in Si solar modules over an entire day and the seasons. Additionally, the HS-NOA63 film can be also very useful as a PAR layer of transparent covers or substrates in other solar power systems such as organic and dye-sensitized solar cells because it has the superior light-harvesting (i.e., AR and light scattering) property over wide ranges of wavelengths $(\lambda=400-1100 \mathrm{~nm})$ and incident angles $\left(0-70^{\circ}\right) .^{24,45,46}$

\section{Surface wettability and self-cleaning behaviour}

In external outdoor environments, accumulations of airborne dust or particles on the surface of solar power systems can lead to the light-absorption degradation of solar cells. Therefore, it is essential to employ the self-cleaning functioned PAR layer into solar power systems. Fig. 7 shows (a) the photographs of a water droplet for the bare PET and HS-NOA63:PET and (b) the sequential photographs for a self-cleaning behaviour of the $\mathrm{Si}$ solar module with the HS-NOA63:PET by rolling down water droplets. In Fig. 7(a), the PET had a hydrophilic surface with a water contact angle (WCA) value of $\sim 75^{\circ}$. The dust particles or contaminants on this hydrophilic surface can be usually just 


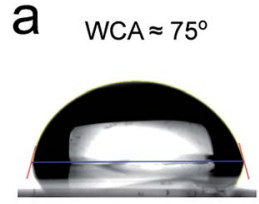

PET

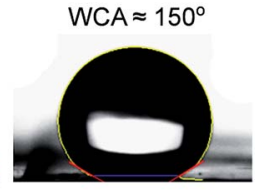

HS-NOA63:PET b

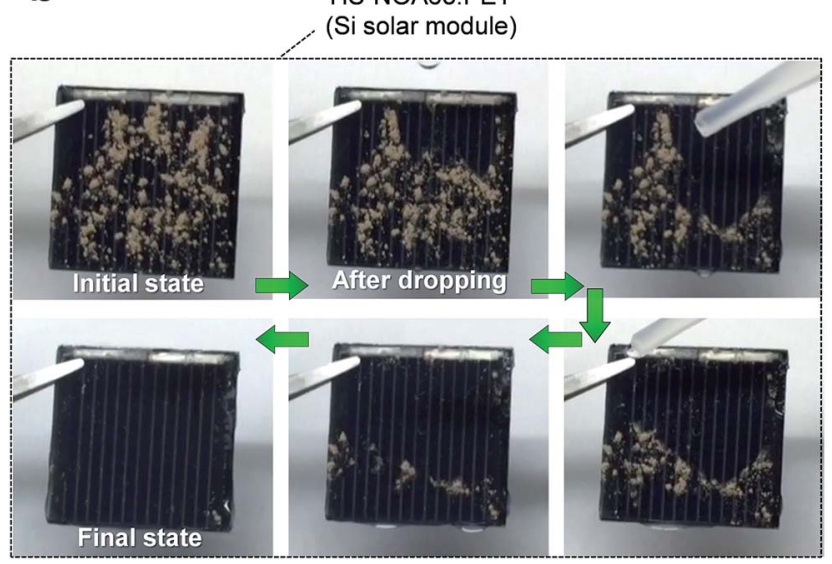

Fig. 7 (a) Photographs of a water droplet for the bare PET and HSNOA63:PET. (b) Sequential photographs for a self-cleaning behaviour of the Si solar module with the HS-NOA63:PET by rolling down water droplets.

relocated or partially remained with water droplets at both the surface and edge of solar modules (see Fig. S2 of the ESI $\dagger$ ).$^{30}$ In outdoor use, these remaining water droplets with dusts or contaminants on the surface of solar modules can be an interruption factor of sunlight incidence into the underneath Si solar cells, which results in the deterioration of overall solar power generation. On the other hand, the surface of the HS-NOA63:PET exhibited a high WCA value of $\sim 150^{\circ}$ (e.g., superhydrophobicity). The superhydrophobic surface (i.e., WCA $\geq 150^{\circ}$ ) can be obtained, when the surface roughness of a material is very large, explained by the Cassie and Baxter model. ${ }^{47}$ Here, the F-NOA63:PET with a flat surface structure had a hydrophilic surface with a WCA value of $\sim 72^{\circ}$ (see Fig. S3 of the ESI $\dagger$ ). It has been reported that the superhydrophobic surface efficiently reduces the probability of water condensations and dust accumulations on the surface (i.e., self-cleaning). ${ }^{31-33}$ The selfcleaning behaviour of the superhydrophobic HS-NOA63 film on the Si solar module can be confirmed in Fig. 7(b). When water droplets were dropped onto the surface of the HS-NOA63 film covered by sand dusts, the dusts were nearly washed by rolling down water droplets without any remained sand dusts and water droplets at its surface. Thus, the PAR layer with an additional self-cleaning ability would enhance the practical feasibility of various solar power systems in real outdoor applications.

\section{Conclusions}

In summary, we have demonstrated the improved light absorption of $\mathrm{Si}$ solar modules by integrating the multifunctional HS-NOA63 film into the outer surface of PET cover as a PAR layer. The HS consisting of nanonipples on micropyramidal arrays on the surface of the NOA63 film was pattern-transferred from the HS-Si mold by the soft lithography. The introduction of the HS-NOA63 film as a PAR layer of PET cover into Si solar modules led to the increased PCE compared to the reference Si solar module in the wide incident angles of $0-70^{\circ}$ due to the mainly increased $I_{\mathrm{sc}}$ caused by its superior AR (i.e., $R_{\mathrm{sw}}=6.1 \%$ ) and light-scattering (i.e., $H_{\mathrm{avg}}=90.7 \%$ ) effects in a wavelength range of $380-1100 \mathrm{~nm}$, exhibiting the PCE relative increment percentage of $6.3 \%$ at $0^{\circ}$ (i.e., from 13.46 to $14.3 \%)$. Moreover, its self-cleaning ability caused by the superhydrophobic surface (i.e., WCA $\approx 150^{\circ}$ ) is very helpful to prevent the Si solar power systems from being polluted by water droplets and dust particles in practical outdoor applications. Therefore, the multifunctional HS-NOA63 film, which can be easily realized by a simple and cost-effective manufacturing technology, can provide a promising strategy for boosting the efficiency of various solar power systems and optic components that use transparent substrates or covers such as glasses, plastics, sapphires, and quartzes.

\section{Acknowledgements}

This work was supported by the National Research Foundation of Korea (NRF) grant funded by the Korea government (MSIP) (No. 2013R1A2A2A01068407).

\section{References}

1 J. Yoon, S. Jo, I. S. Chun, I. Jung, H. S. Kim, M. Meitl, E. Menard, X. Li, J. J. Coleman, Y. Paik and J. A. Rogers, Nature, 2010, 465, 329.

2 M. S. White, M. Kaltenbrunner, E. D. Głowacki, K. Gutnichenko, G. Kettlgruber, I. Graz, S. Aazou, C. Ulbricht, D. A. M. Egbe, M. C. Miron, Z. Major, M. C. Scharber, T. Sekitani, T. Someya, S. Bauer and N. S. Sariciftci, Nat. Photonics, 2013, 7, 11.

3 N. Stutzmann, R. H. Friend and H. Sirringhaus, Science, 2003, 299, 1881.

4 F. R. Fan, Z. Q. Tian and Z. L. Wang, Nano Energy, 2012, 1, 328.

5 D. Liu and T. L. Kelly, Nat. Photonics, 2014, 8, 133.

6 Y. Rong, X. Li, Z. Ku, G. Liu, H. Wang, M. Xu, L. Liu, M. Hu, P. Xiang, Z. Zhou, T. Shu and H. Han, Sol. Energy Mater. Sol. Cells, 2012, 105, 148.

7 Y. Wang, N. Lu, H. Xu, G. Shi, M. Xu, X. Lin, H. Li, W. Wang, D. Qi, Y. Lu and L. Chi, Nano Res., 2010, 3, 520.

8 A. Hongsingthong, T. Krajangsang, I. A. Yunaz, S. Miyajima and M. Konagai, Appl. Phys. Express, 2010, 3, 051102.

9 J. W. Leem, Y. M. Song and J. S. Yu, Nanoscale, 2013, 5, 10455. 10 Y. Liu, A. Das, S. Xu, Z. Lin, C. Xu, Z. L. Wang, A. Rohatgi and C. P. Wong, Adv. Energy Mater., 2012, 2, 47.

11 C. H. Ho, D. H. Lien, H. C. Chang, C. A. Lin, C. F. Kang, M. K. Hsing, K. Y. Lai and J. H. He, Nanoscale, 2012, 4, 7346.

12 J. W. Leem, M. S. Kim and J. S. Yu, J. Opt. Soc. Am. B, 2013, 30, 1665. 
13 Y. M. Song, Y. Xie, V. Malyarchuk, J. Xiao, I. Jung, K. J. Choi, Z. Liu, H. Park, C. Lu, R. H. Kim, R. Li, K. B. Crozier, Y. Huang and J. A. Rogers, Nature, 2013, 497, 95.

14 G. M. Whitesides, E. Ostuni, S. Takayama, X. Jiang and D. E. Ingber, Annu. Rev. Biomed. Eng., 2001, 3, 335.

15 T. Bhuvana, B. Kim, X. Yang, H. Shin and E. Kim, Angew. Chem., Int. Ed., 2013, 52, 1180.

16 M. A. Unger, H. P. Chou, T. Thorsen, A. Scherer and S. R. Quake, Science, 2000, 288, 113.

17 S. H. Ahn and L. J. Guo, ACS Nano, 2009, 3, 2304.

18 C. J. Ting, F. Y. Chang, C. F. Chen and C. P. Chou, J. Micromech. Microeng., 2008, 18, 075001.

19 S. M. Kang, N. Ahn, J. W. Lee, M. Choi and N. G. Park, J. Mater. Chem. A, 2014, 2, 20017.

20 H. K. Raut, S. S. Dinachali, Y. C. Loke, R. Ganesan, K. K. Ansah-Antwi, A. Góra, E. H. Khoo, V. A. Ganesh, M. S. M. Saifullah and S. Ramakrishna, ACS Nano, 2015, 9, 1305.

21 D. H. Ko, J. R. Tumbleston, K. J. Henderson, L. E. Euliss, J. M. DeSimone, R. Lopez and E. T. Samulski, Soft Matter, 2011, 7, 6404.

22 D. K. Cai, A. Neyer, R. Kuckuk and H. M. Heise, Opt. Mater., 2008, 30, 1157.

23 M. Aden, A. Roesner and A. Olowinsky, J. Polym. Sci., Part B: Polym. Phys., 2010, 48, 451.

24 S. Y. Heo, J. K. Koh, G. Kang, S. H. Ahn, W. S. Chi, K. Kim and J. H. Kim, Adv. Energy Mater., 2014, 4, 1300632.

25 J. D. Myers, W. Cao, V. Cassidy, S. H. Eom, R. Zhou, L. Yang, W. You and J. Xue, Energy Environ. Sci., 2012, 5, 6900.

26 Norland Optical Adhesive, retrieved 2015, https:// www.norlandprod.com.

27 C. C. Liang, C. H. Lin, T. C. Cheng, J. Shieh and H. H. Lin, Adv. Mater. Interfaces, 2015, 2, 1500030.

28 M. J. Lee, N. Y. Lee, J. R. Lim, J. B. Kim, M. Kim, H. K. Baik and Y. S. Kim, Adv. Mater., 2006, 18, 3115.

29 J. W. Leem, X. Y. Guan, M. Choi and J. S. Yu, Sol. Energy Mater. Sol. Cells, 2015, 134, 45.

30 Y. B. Park, H. Im, M. Im and Y. K. Choi, J. Mater. Chem., 2011, 21, 633.
31 J. Zhu, C. M. Hsu, Z. Yu, S. Fan and Y. Cui, Nano Lett., 2010, 10, 1979.

32 J. Son, S. Kundu, L. K. Verma, M. Sakhuja, A. J. Danner, C. S. Bhatia and H. Yang, Sol. Energy Mater. Sol. Cells, 2012, 98, 46.

33 C. H. Liu, P. L. Niu and C. K. Sung, J. Phys. D: Appl. Phys., 2014, 47, 015401.

34 R. Kleinhemple, A. Wahl and R. Thielsch, Surf. Coat. Technol., 2011, 205, S502.

35 B. G. Kum, Y. C. Park, Y. J. Chang, J. Y. Jeon and H. M. Jang, Thin Solid Films, 2011, 519, 3778.

36 C. Kim, J. W. Choi, S. Choi, S. Kim, H. Park, H. Song, S. S. Yoon, J. Y. Huh, Y. Kang, H. S. Lee and D. Kim, Sci. Rep., 2016, 6, 21553.

37 D. Qi, N. Lu, H. Xu, B. Yang, C. Huang, M. Xu, L. Gao, Z. Wang and L. Chi, Langmuir, 2009, 25, 7769.

38 C. I. Yeo, Y. M. Song, S. J. Jang and Y. T. Lee, Opt. Express, 2011, 19, A1109.

39 J. W. Leem, D. H. Joo and J. S. Yu, Sol. Energy Mater. Sol. Cells, 2011, 95, 2221.

40 E. Hecht, Optics, Addison-Wesley, NY, USA, 4th edn, 2002, ch. 10.

41 Y. M. Song, H. J. Choi, J. S. Yu and Y. T. Lee, Opt. Express, 2010, 18, 13063.

42 H. Cui, S. Pillai, P. Campbell and M. Green, Sol. Energy Mater. Sol. Cells, 2013, 109, 233.

43 J. Zhao and M. A. Green, IEEE Trans. Electron Devices, 1991, 38, 1925.

44 Y. H. Ko and J. S. Yu, Opt. Express, 2011, 19, 15574.

45 J. D. Myers, W. Cao, V. Cassidy, S. H. Eom, R. Zhou, L. Yang, W. You and J. Xue, Energy Environ. Sci., 2012, 5, 6900.

46 S. Nam, M. Song, D. H. Kim, B. Cho, H. M. Lee, J. D. Kwon, S. G. Park, K. S. Nam, Y. Jeong, S. H. Kwon, Y. C. Park, S. H. Jin, J. W. Kang, S. Jo and C. S. Kim, Sci. Rep., 2014, 4, 4788.

47 A. B. D. Cassie and S. Baxter, Trans. Faraday Soc., 1944, 40, 546. 